\title{
A Chemical Hemostatic Technique for Bleeding from Malignant Wounds
}

\author{
Masaki Kakimoto, M.D., Ph.D., ${ }^{1}$ Hiromi Tokita, M.D., Ph.D., \\ Takashi Okamura, M.D., Ph.D., and Koji Yoshino, M.D. ${ }^{2}$
}

\begin{abstract}
Background: Breast cancer, skin cancer, and head and neck cancer often develops a hemorrhagic malignant wound. Bleeding from the tumor impairs patients' quality of life and can be life threatening, while surgical or electrical hemostasis is often unsuccessful because of the tumor's friability.

Methods: We performed a chemical hemostatic treatment for breast cancer hemorrhage with zinc chloride paste (Mohs' paste), which is usually applied as a fixative in micrographic surgery for cutaneous neoplasms. Five patients with bleeding from breast cancer under various circumstances were treated with this technique in 2008. Results: The method was successful on first application for all five patients, and hemostasis was maintained long term.

Conclusions: This simple technique is effective for bleeding from malignant wounds and should be learned by health professionals performing cancer care.
\end{abstract}

\section{Introduction}

$\mathbf{M}$ ALIGNANT WOUNDS from primary or metastatic carcinoma are generally incurable even after surgical or radiologic treatment. They are managed with palliative methods to lessen symptoms such as pain, exudate, and bleeding. ${ }^{1-3}$ Bleeding from malignant wounds impairs patients' quality of life and can be life threatening on occasion.

Although hydropolymer dressings or solutions of antiprotozoal drugs are used in an attempt to improve symptoms, they are not sufficient to control bleeding. ${ }^{4-6}$ Breast cancer demonstrates the greatest propensity to develop malignant wounds, with approximately $50 \%$ of such wounds associated with this diagnosis. 7,8

While he was a medical student in the 1930s, Frederic E. Mohs developed a technique for chemical fixation of a cutaneous tumor followed by serial excision. He published the results of this "chemosurgery technique" in 1941.9,10 The fixative, Mohs' paste, which contains zinc chloride, is mainly used for micrographic excision of cutaneous carcinomas. In this technique the paste is generally applied to the tumor 24 hours before excision. ${ }^{11}$ The physician then performs a sequential series of excisions until no residual tumor can be found in the bottom layer of the specimen by microscopic examination. The tissue to be excised is numbed, so local anesthetic is not necessary, and the operative field is bloodless as a result of chemical infiltration and fixation. ${ }^{11,12}$ Utilizing the same effect, hemostasis of bleeding from a carcinoma can be rapidly achieved by direct application of the paste without performing any surgical procedure. We apply Mohs' paste for malignant wound hemorrhage from breast cancer, which is common in our daily medical care.

\section{Method}

\section{Patients}

In 2008 we performed chemical hemostatic treatment for five patients with malignant wounds from breast cancer. Four were primary tumors and the other was a metastasis on the skin of the arm. Patient characteristics and time to next treatment are shown in Table 1. Patients underwent various pretreatments such as surgery, chemotherapy, radiotherapy, and hormonal therapy. Case 2-4 had given up care at home due to frequent bleeding. Case 1 and Case 4 needed blood transfusions before the hemostatic treatment. Case 1 was undergoing EC (epirubicin + cyclophospamide) chemotherapy. Although the tumor had partially responded to the therapy and had shrunk $50 \%$ in diameter, sudden massive bleeding occurred, leading to hemorrhagic shock.

\section{Preparing Mohs' paste}

The ingredients for Mohs' paste are shown in Table 2. The fixative preparation is designed to release the zinc chloride in a

\footnotetext{
${ }^{1}$ Department of Surgery, Tokyo Metropolitan Ohtsuka Hospital, Tokyo, Japan.

${ }^{2}$ Department of Dermatology, Tokyo Metropolitan Komagome Hospital, Tokyo, Japan.

Accepted August 10, 2009.
} 
Table 1. Patient Characteristics

\begin{tabular}{ccllrr}
\hline Case & $\begin{array}{c}\text { Age (years) } \\
\text { (all female) }\end{array}$ & Primary or metastasis & \multicolumn{1}{c}{$\begin{array}{c}\text { Size of } \\
\text { Pretreatment }\end{array}$} & $\begin{array}{c}\text { Time to next } \\
\text { treatment (days) }\end{array}$ \\
\hline 1 & 82 & Primary & Hormonal therapy chemotherapy & $8 \times 12$ & 21 \\
2 & 88 & Primary & Radiation & $30 \times 20$ & $7 \times 5$ \\
3 & 61 & Metastasis of arm skin & Surgery chemotherapy & $15 \times 15$ & 48 \\
4 & 64 & Primary & None & $3 \times 3$ & 90 \\
5 & 75 & Primary & Hormonal therapy & 90 \\
\hline
\end{tabular}

controllable manner. ${ }^{2-6}$ First, distilled water and zinc chloride are put in a beaker and stirred until homogenized $(50 \mathrm{~mL}$ saturated solution of zinc chloride).

The mixture is allowed to cool to room temperature (it will have generated heat of dissolution). In a mortar, zinc powder is then dissolved little by little in the solution, to avoid lumps.

Glycerin is added to adjust the viscosity of the paste $(110 \mathrm{~g}$ Mohs' paste made up). Sanguinaria canadensis (bloodroot), which was originally contained as a binder, ${ }^{12}$ is omissible in our composition. No sterilization is needed; the paste can be stored in a plastic ointment jar for 1 year at room temperature.

The paste should be handled with plastic gloves, and the skin should be washed with water to prevent a sore if the paste contacts the skin.

\section{Technique}

We advise administering simple analgesics before the procedure because penetration of the fixative may cause some pain. Preliminary application of lidocaine jelly is also effective. Although Mohs' paste dissolves easily in water, when it is first made up the paste is thick so that we use two wooden tongue depressors for application (Fig. 1). Surround the tumor with waterproof film and cover normal skin with cotton gauze before application (Fig. 2). The paste will soften and flow out, to rapidly contact blood and exudate from the malignant wound. We paint Mohs' paste directly on the tumor or paint it on gauze first and use this to apply pressure on the tumor when there are active bleeding sites. The desired depth of penetration is determined by the time the paste is left on the tumor. For skin cancer chemosurgery the time ranges from a few minutes to 48 hours $^{12}$; however, it takes only 5 or 10 minutes to stop oozing from breast cancer. The extra paste is wiped off with tap water-soaked gauze to finish the process. Most surface bleeding points can be controlled with this brief treatment (Fig. 3). The surface of the malignant wound becomes dry and hard after a few hours.

\section{Results}

The hemostatic treatment was successful on first application in all five patients. Active bleeding from arteries had been stopped by applying pressure for 10 minutes after applica-

Table 2. Ingredients of Mohs' Paste

\begin{tabular}{lll}
\hline Zinc chloride & $60 \mathrm{~g}$ & $1200 \mathrm{~g}$ \\
Distilled water & $30 \mathrm{~mL}$ & $600 \mathrm{~mL}$ \\
Zinc powder & $30 \mathrm{~g}$ & $600 \mathrm{~g}$ \\
Glycerin & $15 \mathrm{~mL}$ & $300 \mathrm{~mL}$ \\
End product & $110 \mathrm{~g}$ & $2200 \mathrm{~g}$ \\
\hline
\end{tabular}

tion. Bleeding control was maintained from 3 weeks to more than 3 months. In all cases, either the patients or their families learned to change wound dressings by themselves after the treatment. Odor and exudate were also reduced.

\section{Discussion}

Although Mohs' paste was invented approximately 70 years ago, it remains important for micrographic surgery of cutaneous carcinomas and can maximize the amount of normal tissue preserved without remnant tumor. ${ }^{14,15}$ Currently, however, this chemosurgical technique is not necessarily the first-line choice for skin tumor resection because of the extra costs incurred in repeating surgery and histologic examination. Hence many health professionals are unaware of the strong hemostatic effect of this preparation on hemorrhage from neoplasm.

Breast cancer is the malignancy most likely to develop malignant wounds. ${ }^{7}$ Bleeding from breast cancer can occur in palliative care patients with terminal stage disease, but also during neoadjuvant chemotherapy or radiation therapy for advanced cancer with skin invasion. Suturing or electric coagulation have poor rates of successful hemostasis and can even cause further bleeding because the malignant wounds of breast cancer tend to be friable and contain many abnormal small vessels. The present chemical hemostatic technique with zinc chloride paste is useful for physicians, surgeons, oncologists, and palliative care nurses. This treatment is also effective for odor and exudate from malignant wound, and therefore contributes to patients' quality of life. Later appli-

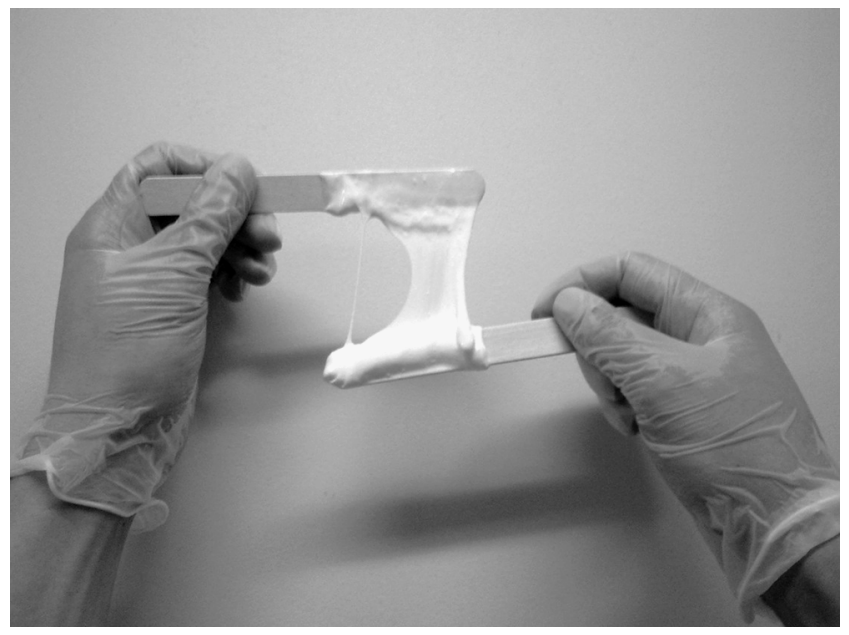

FIG. 1. Two wooden tongue depressors are used to apply the paste. 


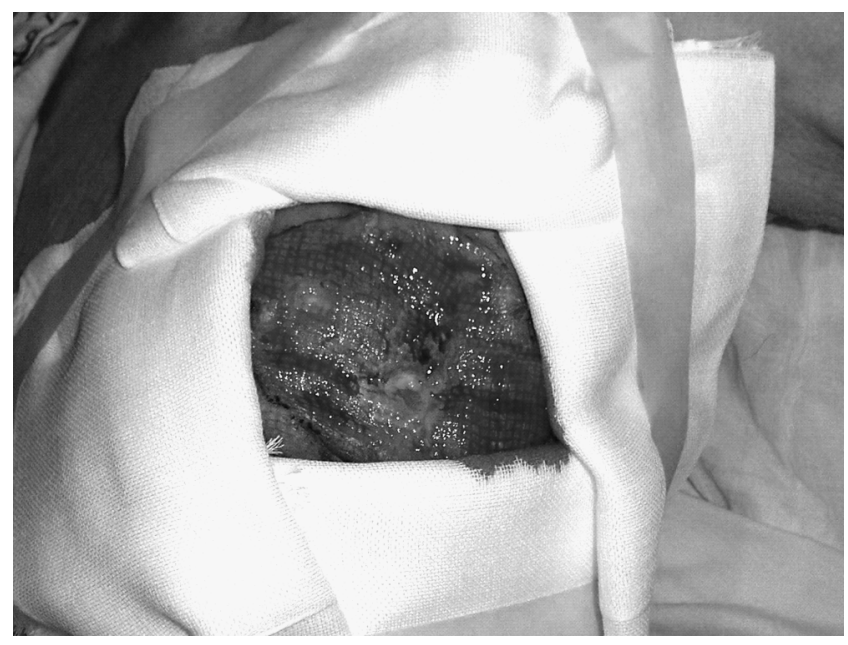

FIG. 2. The tumor is surrounded with waterproof film and cotton gauze covers normal skin before application. (The checked appearance of the wound surface is caused by the imprint of an overlying dressing, now removed.)

cation of metronidazole powder is helpful for more complete deodorization. ${ }^{15}$

With repeated deeper fixation and sharp resection, palliative debulking surgery for unresectable breast cancer can be performed. However, excessive penetration of the chest wall may cause complications such as pleural perforation, and such resection should therefore cease when the pectoral muscle layer is exposed.

\section{Acknowledgments}

The authors acknowledge members of Department of Dermatology, Tokyo Metropolitan Komagome Hospital and palliative care team, Tokyo Metropolitan Ohtsuka Hospital.

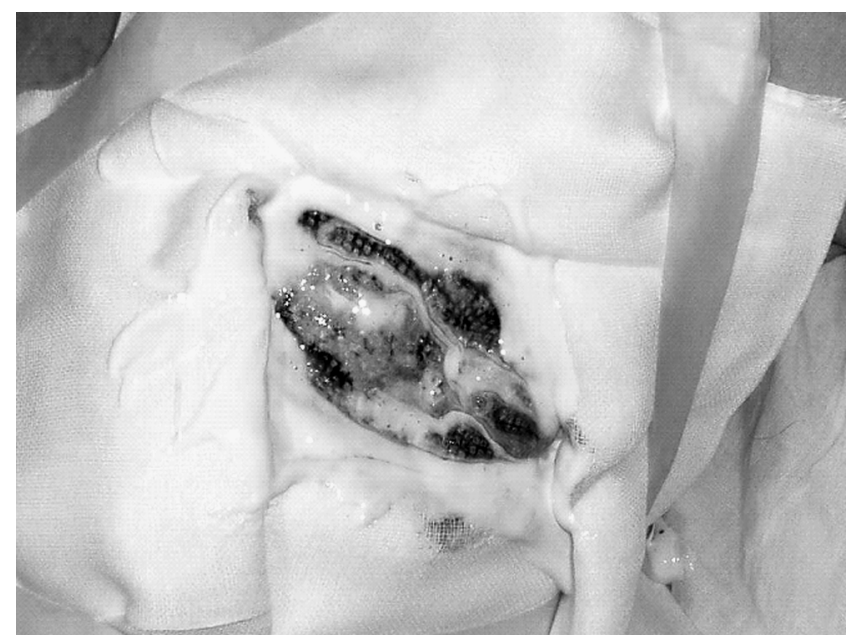

FIG. 3. The excess paste is wiped off with tap water-soaked gauze. Hemostasis is achieved.

\section{Author Disclosure Statement}

No competing financial interests exist.

\section{References}

1. McDonald A, Lesage P: Palliative management of pressure ulcers and malignant wounds in patients with advanced illness. J Palliat Med 2006;9:285-295.

2. Adderley U, Smith R: Topical agents and dressings for fungating wounds. Cochrane Database Syst Rev 2007;18: CD003948.

3. Schiech L: Malignant cutaneous wounds. Clin J Oncol Nurs 2002;6:305-309.

4. Lund-Nielsen B, Müller K, Adamsen L: Qualitative and quantitative evaluation of a new regimen for malignant wounds in women with advanced breast cancer. J Wound Care 2005;14:69-73.

5. Wilson V: Assessment and management of fungating wounds: A review. Br J Community Nurs 2005;10:S28-34.

6. Naylor W: Using a new foam dressing in the care of fungating wounds. Br J Nurs 2001;10(6 Suppl):S24-30.

7. Maida V, Ennis M, Kuziemsky C, Trozzolo L: Symptoms associated with malignant wounds: A prospective case series. J Pain Symptom Manage 2009;37:206-211.

8. Schulz V, Triska $\mathrm{OH}$, Tonkin $\mathrm{K}$ : Malignant wounds: Caregiver-determined clinical problems. J Pain Symptom Manage 2002;24:572-577.

9. Mohs FE, Sevringhaus EL, Schmidt ER: Conservative amputation of gangrenous parts by chemosurgery. Ann Surg 1941;114:274-282.

10. Swanson NA. Mohs surgery: Technique, indications, applications, and the future. Arch Dermatol 1983;119:761-773.

11. Levine H: Cutaneous carcinoma of the head and neck: Management of massive and previously uncontrolled lesions. Laryngoscope 1983;93:87-105.

12. Mohs FE: Chemosurgery. Clin Plast Surg 1980;7:349-360.

13. Albertini JG: Mohs micrographic surgery pearls. Semin Cutan Med Surg 2004;23:184-195.

14. Turner RJ, Leonard N, Malcolm AJ, Lawrence CM, Dahl MG: A retrospective study of outcome of Mohs' micrographic surgery for cutaneous squamous cell carcinoma using formalin fixed sections. Br J Dermatol 2000;142:752-757.

15. Paul JC, Pieper BA: Topical metronidazole for the treatment of wound odor: A review of the literature. Ostomy Wound Manage 2008;54:18-27; quiz 28-29.

Address correspondence to: Masaki Kakimoto, M.D., Ph.D. Department of Surgery Tokyo Metropolitan Ohtsuka Hospital 2-8-1 Minami-Otsuka Toshima-ku Tokyo Japan

E-mail: m-kakimot-o@ohtsuka-hospital.toshima.tokyo.jp 
